\title{
Knockdown of long non-coding RNA PVT1 inhibits the proliferation of Raji cells through cell cycle regulation
}

\author{
CHANLI ZHENG ${ }^{1}$, YU XIAO ${ }^{1}$, YANGQIU LI ${ }^{1,2}$ and DONGMEI HE ${ }^{1}$ \\ ${ }^{1}$ Institute of Hematology, School of Medicine; ${ }^{2}$ Key Laboratory for Regenerative Medicine of Ministry of Education, \\ College of Life Science and Technology, Jinan University, Guangzhou, Guangdong 510632, P.R. China
}

Received October 11, 2018; Accepted February 20, 2019

DOI: $10.3892 / \mathrm{ol} .2019 .10450$

\begin{abstract}
Long non-coding RNA plasmacytoma variant translocation 1 ( $P V T 1)$ has been reported to be associated with oncogenesis. However, the functional role of $P V T 1$ in Burkitt lymphoma has not yet been addressed. The purpose of the present study was to investigate the effect of PVTI knockdown by small interfering RNA (siRNA) on the proliferation of Burkitt lymphoma Raji cells and to explore its possible mechanism of action. An effective siRNA targeting PVT1 was screened and the corresponding short hairpin RNA (shRNA) was reconstructed into a lentiviral vector. Cell proliferation and cell cycle distribution were assessed by Cell Counting kit- 8 assay and flow cytometry, respectively. Protein expression levels of c-Myc, cyclin-dependent kinase inhibitor1A (CDKN1A, P21) and cyclin E1 (CCNE1) were detected by western blotting. A polymerase chain reaction (PCR) array was used to analyse the expression of genes associated with the cell cycle. PVT1 knockdown markedly suppressed proliferation, and induced cell cycle arrest at the $G_{0} / G_{1}$ phase in Raji cells. Protein expression levels of c-Myc and CCNE1 were reduced, whereas $\mathrm{P} 21$ protein expression was markedly increased following downregulation of PVT1 in Raji cells. The cell cycle PCR array revealed that 54 genes were upregulated and 26 genes were downregulated in Raji cells following PVT1 knockdown. Reverse transcription-quantitative PCR demonstrated that cyclin G2 (CCNG2), CDKN1A, Retinoblastoma-like 2 (RBL2, p130), HUS1 checkpoint homolog, cyclin dependent kinase inhibitor $3(C D K N 3)$ and cyclin dependent kinase inhibitor 1B $(C D K N 1 B)$ expression were upregulated, whereas the expression levels of $C C N E 1$, cyclin D1 (CCNDI) and cell division cycle $20(C D C 20)$ were downregulated in Raji cells with $P V T 1$ knockdown. In conclusion, $P V T 1$ knockdown may inhibit the proliferation of
\end{abstract}

Correspondence to: Professor Dongmei He, Institute of Hematology, School of Medicine, Jinan University, 601 HuangPu Da Dao Xi, Guangzhou, Guangdong 510632, P.R. China

E-mail: thedm@jnu.edu.cn

Key words: long non-coding RNA, plasmacytoma variant translocation 1, Raji cells, proliferation, cell cycle
Raji cells by arresting cells in $\mathrm{G}_{0} / \mathrm{G}_{1}$ phase. Furthermore, inhibition of cell proliferation may be associated with a reduction inc-Myc expression and alterations in the expression levels of cell cycle-associated genes.

\section{Introduction}

Burkitt lymphoma (BL) is an aggressive form of B-cell lymphoma that mostly affects children and adolescents. In $\mathrm{BL}$, dysregulation of the oncogenic transcription factor Myc is considered to be the major driving force of lymphoma development $(1,2)$. However, the molecular mechanisms of the pathogenesis of BL have not been fully elucidated.

Long non-coding RNAs (lncRNAs) are defined as cellular RNA molecules, $>200$ base pairs in length, without protein-coding capacity, which act as key regulators at transcriptional and post-transcriptional levels $(3,4)$. Increasing evidence indicates that lncRNAs are involved in various biological processes, including DNA replication, stem cell pluripotency, proliferation and apoptosis $(4,5)$. LncRNAs serve a critical role in the development of various types of human cancer, including haematopoietic malignancies, and may be potential targets for tumour treatment (5).

Human plasmacytoma variant translocation 1 ( $P V T 1)$, also known as the Pvtl oncogene, is a lncRNA that is homologous to the mouse plasmacytoma variant translocation gene. PVT1 is located on chromosome $8, \sim 55 \mathrm{~kb}$ distal to the MYC proto-oncogene bHLH transcription factor $(c-M y c)$ gene, and is frequently involved in translocations that occur in variant BL $(6,7)$. The overexpression of PVT1 is one of the most frequent events in a variety of malignant diseases, including melanoma (8), hepatocellular carcinoma $(9,10)$, thyroid cancer and colorectal cancer $(11,12)$. A number of studies have demonstrated that lncRNA PVT1 interacts with the proliferation-associated nucleolar proteins NOP2 or c-Myc, stabilizes these proteins against degradation, and negatively modulates microRNA (miRNA) as a competing endogenous RNA or a molecular sponge, in order to exert a tumour-promoting effect $(8,10,13,14)$. A large genome-wide association study identified one high-risk single nucleotide polymorphism (SNP; rs2608053) for classic Hodgkin lymphoma at 8q24 near the $M y c / P V T 1$ locus, which is associated with patient outcome (15). In a meta-analysis, two independent SNPs, rs13255292 and rs4733601, at 8q24.21 were identified for 
diffuse large B cell lymphoma (16). However, the functional role and molecular mechanism of $P V T 1$ in BL remain unclear.

In the present study, knockdown of $P V T 1$ was able to inhibit Raji cell growth by regulating cell cycle progression. Furthermore, it was revealed that $P V T 1$ may serve an important role in $\mathrm{G}_{0} / \mathrm{G}_{1}$ arrest, which may be associated with the expression of $c-M y c$ and cell cycle-associated genes. Together, these results indicated that IncRNA PVTI may serve a critical role in Raji cell proliferation, and may be considered a candidate target for novel treatment of human BL.

\section{Materials and methods}

Cell culture and transfection. The Raji cell line was purchased from the Cell Bank of Type Culture Collection of Chinese Academy of Sciences (Shanghai, China. http://www. cellbank. org. cn/index. asp). Cells were cultured in RPMI-1640 medium (Gibco; Thermo Fisher Scientific, Inc., Waltham, MA, USA), supplemented with $10 \%$ heat-inactivated foetal bovine serum (FBS; Gibco; Thermo Fisher Scientific, Inc.) at $37^{\circ} \mathrm{C}$ in a humidified incubator with $5 \% \mathrm{CO}_{2}$. Four small interfering RNA (siRNA) sequences targeting PVT1 (siRNA54, siRNA176, siRNA845, siRNA1055) and a scrambled control (SC) siRNA were designed and synthesized by Shanghai GenePharma Co. , Ltd. (Shanghai, China). The sequences of the siRNA are as follows: $P V T 1$-siRNA54: 5'-CCUGAUGGAUUUACA GUGATT-3', $P V T 1$-siRNA176: 5'-GCUGAAUGCCUCAUG GAUUTT-3', $P V T 1$-siRNA845: 5'-CCUGUUACACCUGGG AUUUTT-3', PVT1-siRNA1055: 5'-GCUUCUCCUGUUGCU GCUATT-3', SC-siRNA: 5'-GCUACGAUCUGCCUAAGA UTT-3'. Raji cells ( $\left.3-4 \times 10^{5} \mathrm{cells} / \mathrm{ml}\right)$ in the exponential growth phase were grown for $24 \mathrm{~h}$, then $P V T 1$-siRNA54, -siRNA176, -siRNA845 and-siRNA1055 were transfected into Raji cells using HiPerfect (Qiagen, Inc., Valencia, CA, USA) according to the manufacturer's protocol. In addition to non-silencing SC siRNA control, cells in the mock transfection group were treated with HiPerfect agents only. The total concentration of siRNA applied in eachcase was $100 \mathrm{nM}$. At 24 and $48 \mathrm{~h}$ post-transfection, silencing of $P V T l$ RNA was examined.

Reverse transcription-quantitative polymerase chain reaction $(R T-q P C R)$. Total RNA from the Raji cells of post-transfection was isolated using a TRIzol ${ }^{\circledR}$ total RNA isolation system (Invitrogen; Thermo Fisher Scientific, Inc.). RNA purity and concentration were measured using a spectrophotometer, and RNA was reverse transcribed into first-strand cDNA using random hexamer primers and the reverse transcriptase Superscript II kit (Toyobo Life Science, Osaka, Japan), according to the manufacturer's protocol. The $2^{-\Delta \mathrm{Ct}}$ method (17) was used to analyse the relative changes in gene expression in RT-qPCR experiments with SYBR Green (Toyobo Life Science, Japan). The primers were designed and synthesized by Shanghai GeneChem Co., Ltd. (Shanghai, China). The primer sequences are listed in Table I. GAPDH was used as a reference gene. The total PCR reaction volume was $20 \mu 1$ and reaction conditions were as follows: Enzyme activation at $95^{\circ} \mathrm{C}$ for $10 \mathrm{~min}$, followed by 40 cycles at $95^{\circ} \mathrm{C}$ for $15 \mathrm{sec}, 60^{\circ} \mathrm{C}$ for $15 \mathrm{sec}$ and $72^{\circ} \mathrm{C}$ for $32 \mathrm{sec}$. At the end of each run a melting curve was performed, starting at $65^{\circ} \mathrm{C}$ and reaching $95^{\circ} \mathrm{C}$ with an increase of $1^{\circ} \mathrm{C} / 2 \mathrm{sec}$, to verify primer specificities, specificity of amplification and absence of primer dimers. RT-qPCR was repeated in at least three separate experiments.

Western blot analysis. Cells were washed with PBS (10 mM, $\mathrm{pH}$ 7.4) and incubated in $200 \mu 1$ cell lysis buffer (Beyotime Institute of Biotechnology) on ice for $30 \mathrm{~min}$, and centrifuged at $13,000 \mathrm{x}$ g for $15 \mathrm{~min}$ at $4^{\circ} \mathrm{C}$. The protein content of the cell lysate was determined by Bio-Rad protein assay (Bio-Rad Laboratories, Inc., Hercules, CA, USA), according to the manufacturer's protocol. Whole cell extracts equivalent to $100 \mu \mathrm{g}$ total protein were separated by $8 \%$ SDS-PAGE and transferred to nitrocellulose membranes (Gibco; Thermo Fisher Scientific, Inc.) at $18 \mathrm{~V}$ for $10-15 \mathrm{~min}$. The blots were immersed in blocking buffer (10\% non-fat dry milk, $1 \%$ Tween-20, $20 \mathrm{mM}$ Tris-buffered saline, $\mathrm{pH} \mathrm{7.5)}$ for $1 \mathrm{~h}$ at room temperature, and were then incubated with appropriate anti-human primary antibodies [rabbit immunoglobulin $\mathrm{G}(\mathrm{IgG})$ anti-c-Myc (1:1,000 dilution; cat. no., sc-40, Santa Cruz Biotechnology, Inc., Dallas, TX, USA), anti-P21 (1:1,000 dilution; cat. no., ab109520, Abcam, Cambridge, MA, USA) or anti-cyclin E1 (CCNE1) (1:1,000 dilution; cat. no., ab33911, Abcam, Cambridge, MA, USA USA), or mouse anti-GAPDH/IgG (1:1,000 dilution; cat. no., ab8245, Abcam, Cambridge, MA, USA) USA] in blocking buffer overnight at $4^{\circ} \mathrm{C}$. Blots were then incubated with anti-rabbit (cat. no., sc-2357) or anti-mouse (cat. no., sc-516102) horseradish peroxidase-conjugated secondary antibodies (1:2,000 dilution; Santa Cruz Biotechnology, Inc.) for $1 \mathrm{~h}$ at room temperature and bands were detected by chemiluminescence using Enhanced Chemiluminescence Hyperfilm (EMD, Millpore, USA).

PVT1 short hairpinRNA (shRNA)-expressing plasmid construction and cloning screen. To stably knockdown the expression of $P V T 1$, a shRNA sequence targeting PVT1 (siRNA1055) was cloned into the pGV248-lentivirus vector (Shanghai GenePharma Co., Ltd.). Subsequently, PVTl knockdown vectors were reconstructed and sequenced. pGV248 vector containing the negative control (NC) shRNA was used as a control. Subsequently, 293T cells from the Cell Bank of Type Culture Collection of Chinese Academy of Sciences (Shanghai, China) were cultured in Dulbecco's modified Eagle's medium (Gibco; ThermoFisher Scientific, Inc., Waltham, MA, USA) containing $10 \% \mathrm{FBS}$, maintained at $37^{\circ} \mathrm{C}$ in a humidified incubator with $5 \% \mathrm{CO}_{2}$ and transfected with pGV248-shRNAs, Helper 1.0 and Helper 2.0 (Shanghai GenePharma Co., Ltd.) using Lipofectamine ${ }^{\circledR} 2000$ reagent (Invitrogen; Thermo Fisher Scientific, Inc.). The media were replaced with $10 \mathrm{ml}$ fresh medium after incubation overnight. The virus-containing supernatants (LV-PVTI-shRNA and LV-NC-shRNA) were collected after $48 \mathrm{~h}$. Raji cells were infected, respectively, with LV-PVTI-shRNA and LV-NC-shRNA (multiplicity of infection $=250$ ) at $37^{\circ} \mathrm{C}$ for $72 \mathrm{~h}$, and were then selected using $4 \mu \mathrm{g} / \mathrm{ml}$ puromycin to screen single cell clones for 2 weeks and expand the culture for 4 weeks. Green fluorescence of cells was observed by inverted fluorescence microscopy. The knockdown efficiency was measured using RT-qPCR. Cell proliferation and cell cycle distribution were analysed in positive clone Raji cells. In subsequent assays, Raji cells were divided into three groups: Blank control group (cells without infection), NC group (cells with LV-NC-shRNA) and PVTI knockdown group (cells with LV-PVT1-shRNA). 
Table I. Sequences of primers used for reverse transcription-quantitative polymerase chain reaction.

\begin{tabular}{|c|c|c|}
\hline Gene & Forward primer (5'-3') & Reverse primer $\left(5^{\prime}-3^{\prime}\right)$ \\
\hline$P V T 1$ & GTCTTGGTGCTCTGTGTTC & CCCGTTATTCTGTCCTTCT \\
\hline CCNG2 & GGTTTCACCTTCATAAGAGCC & GCTGAGTTTGATTGAGGCTAC \\
\hline$C D K N 1 A$ & AGCGACCTTCCTCATCCACC & AAGACAACTACTCCCAGCCCCATA \\
\hline HUS1 & ATGGGTCACAATGCGGCTACT & GCTAACATCGGAAAACTTATCTCG \\
\hline$C D K N 1 B$ & GGGCAAGTACGAGTGGCAAGAG & CAAATGCGTGTCCTCAGAGTTAGC \\
\hline$C D K N 3$ & AGTCCCAAACCTTCTGGATCTCTAC & CTCCCAAGTCCTCCATAGCAGTG \\
\hline$R B L 2$ & TTCTGGTAGTGCTGGCTGGTG & GGGTGACTGAAGTTCGTGCTG \\
\hline CCNE1 & AAAGGTTTCAGGGTATCAGTGGTG & TCTCTGTGGGTCTGTATGTTGTGTG \\
\hline CCNDI & CCCTCGGTGTCCTACTTCAAATGT & GGAAGCGGTCCAGGTAGTTCAT \\
\hline$C D C 20$ & TCACCAGAGCTTGCACTCCAC & ACCTGCCGTTACATTCCTTCC \\
\hline$G A P D H$ & GGACCTGACCTGCCGTCTAG & GTAGCCCAGGATGCCCTTGA \\
\hline
\end{tabular}

Cell proliferation assay. For the quantitative determination of cellular proliferation, a Cell Counting kit-8 (CCK8; Dojindo Molecular Technologies, Inc., Kumamoto, Japan) assay was performed. The assay was performed for Raji cells carrying LV-PVT1-shRNA and LV-NC-shRNA. Cells were seeded at a density of $5 \times 10^{4}$ cells/well in 96-well plates and cultured at $37^{\circ} \mathrm{C}$ with $5 \% \mathrm{CO}_{2}$ in a humidified incubator. According to the manufacturer's protoocols $10 \mu \mathrm{lCCK} 8$ solution was added $4 \mathrm{~h}$ prior to the end of incubation at $37^{\circ} \mathrm{C}$ at $24,48,72$ and $96 \mathrm{~h}$. Cell proliferation was measured using a spectrophotometer (Bio-Rad Laboratories, Inc.) at an absorbance wavelength of $450 \mathrm{~nm}$. All experiments were repeated three times.

Cell cycle assay. Raji cells containing LV-PVT1-shRNA or LV-NC-shRNA were harvested. The cells were fixed with $70 \%$ ethanol at $-20^{\circ} \mathrm{C}$ overnight and stained with propidium iodide (5 $\mu \mathrm{g} / \mathrm{ml}$; Sigma-Aldrich; Merck KGaA, Darmstadt, Germany) in the presence of ribonuclease A $(1 \mathrm{mg} / \mathrm{ml}$; Sigma-Aldrich; Merck KGaA) for $30 \mathrm{~min}$ at room temperature. The cell cycle distribution was analysed via flow cytometry (BD Biosciences, San Jose, CA, USA). All experiments were repeated three times.

RT-PCR-based array analysis. Human Cell Cycle RT ${ }^{2}$ RNA QC PCR arrays (Qiagen, Inc.) were used to screen a panel of 84 representative cell cycle-associated genes in Raji cells infected with LV-PVT1-shRNA. Total RNA was isolated from the LV-PVT1-shRNA and LV-NC-shRNA cells using the Qiagen RNeasy Mini kit (Qiagen, Inc.) according to the manufacturer's protocol. RNA was quantified by the NanoDrop ${ }^{\circledR}$ ND-1000 (NanoDrop; Thermo Scientific, Inc., Wilmington, DE, USA), and quality was assessed by visualizing $18 \mathrm{~S}$ and $28 \mathrm{~S}$ ribosomal RNA bands separated by $1 \%$ agarose gel electrophoresis with ethidium bromide staining (Sigma-Aldrich; Merck KGaA). According to manufacturer's protocol, the first-strand cDNA was obtained using an $\mathrm{RT}^{2}$ First Strand kit (Qiagen, Inc.). qPCR was conducted using 2X RT2 ${ }^{2}$ SYBR Green qPCR Master Mix (Qiagen, Inc.) on a Bio-Rad Real-Time PCR system (Bio-Rad Laboratories, Inc.) according to the $\mathrm{RT}^{2}$ Profiler PCR array protocols under the following conditions: $95^{\circ} \mathrm{C}$ for $10 \mathrm{~min}$, then 40 cycles at $95^{\circ} \mathrm{C}$ for $15 \mathrm{sec}$ and $60^{\circ} \mathrm{C}$ for $1 \mathrm{~min}$. At the end of each run a melting curve was performed, extending at $60^{\circ} \mathrm{C}$ for $1 \mathrm{~min}$. Microarray data were normalized for housekeeping genes ( $A C T B, B 2 M, G A P D H, H P R T 1, R P L P O)$ by calculating the $\Delta \mathrm{Cq}$ and $2^{-\Delta \Delta \mathrm{Cq}}(17)$ for each gene of interest in the plate. Fold changes of each gene between the LV-PVTl-shRNA and LV-NC-shRNA groups were calculated as $2^{-\Delta \Delta C t}$, and scatter plots were analysed.

Statistical analysis. All data are expressed as the means \pm standard deviation. Differences among three or more groups were compared using one-way analysis of variance (ANOVA), followed by SNK post hoc test. All data were analysed using SPSS v13.0 software (SPSS, Inc., Chicago, IL, USA). $\mathrm{P}<0.05$ was considered to indicate a statistically significant difference.

\section{Results}

PVT1 siRNAs or shRNA suppress PVT1 RNA and c-Myc protein expression in Raji cells. To determine the efficiency of PVT1 inhibition following siRNA (siRNA54, siRNA176, siRNA845, siRNA1055) transfection, PVT1 RNA expression was analysed. RT-qPCR data were obtained from at least three independent experiments. The relative qPCR formula: $2^{-\Delta \mathrm{Ct}}$ x $100 \%$ was used. As shown in Fig. 1A, at 24 and $48 \mathrm{~h}$ after transient transfection, the relative expression levels of $P V T 1$ RNA in Raji cells transfected with PVT1 siRNA (siRNA1055) were significantly lower than in cells transfected with the control SC siRNA ( $\mathrm{P}<0.05)$. Conversely, siRNA54, siRNA176, siRNA845 had no significant effect on the expression of PVT1 RNA when compared respectively with the control SC siRNA. A specific siRNA (siRNA1055) against PVT1 was used to stably knockdown PVT1 expression. Subsequently, lentiviral vectors carrying LV-PVT1-shRNA or LV-NC-shRNA were successfully constructed. Raji cells stably carrying $P V T 1$-shRNA or NC-shRNA were screened. Raji cells expressing LV-PVT1-shRNA were observed to exhibit green fluorescence under an inverted fluorescence microscope (data not shown). As shown in Fig. 1B, following stable infection with the LV-PVT1-shRNA expression vector, the expression levels of PVT1 RNA were significantly reduced compared with in the LV-NC-shRNA and blank control cell groups 

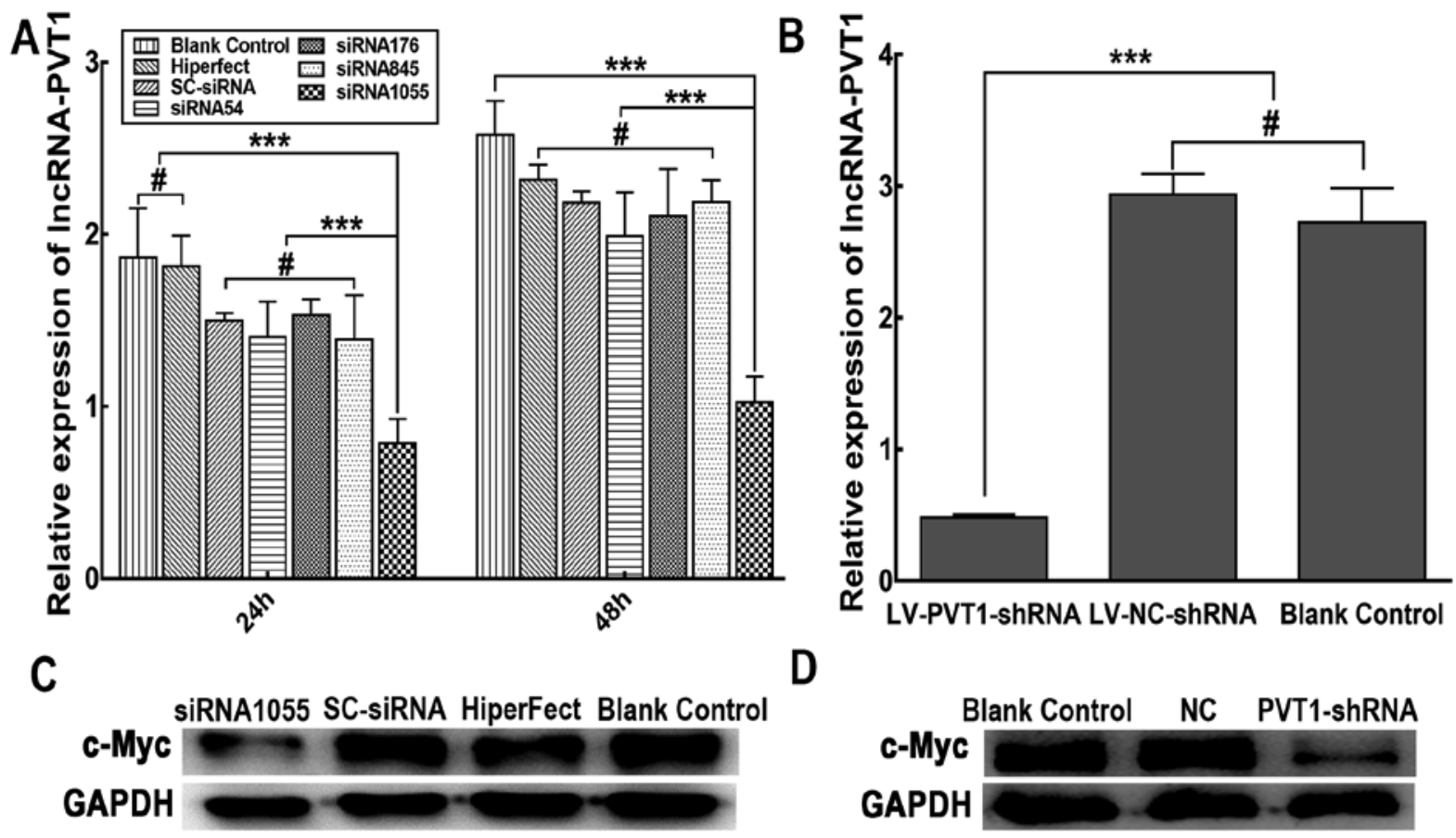

\section{D}

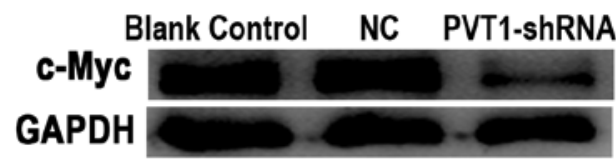

Figure 1. Effect of $P V T 1$ siRNAs or shRNA on $P V T 1$ RNA and c-Myc protein expression in Raji cells. (A) Suppression of $P V T 1$ RNA expression was measured by reverse transcription-quantitative polymerase chain reaction at 24 and $48 \mathrm{~h}$ after transient transfection with PVT1 siRNAs (siRNA54, siRNA176, siRNA845 and siRNA1055). Non-silencing SC siRNA-transfected, mock-transfected (HiPerfect reagents only) and non-treated (blank control) cells were used as controls. GAPDH was used as the reference gene. (B) Expression levels of PVT1 RNA in Raji cells stably infected with LV-PVT1-shRNA and LV-NC-shRNA. (C) Expression of c-Myc protein in Raji cells at $48 \mathrm{~h}$ after $P V T 1$-siRNA transfection, assessed by western blotting. (D) Protein expression levels of c-Myc in Raji cells stably infected with LV-PVT1-shRNA. Representative images are shown. The results are expressed as the mean values of three independent experiments \pm standard deviation. ${ }^{* * * *} \mathrm{P}<0.01,{ }^{\#} \mathrm{P}>0.05$. c-Myc, MYC proto-oncogene bHLH transcription factor; IncRNA, long non-coding RNA; LV, lentiviral vector; NC, negative control; PVT1, plasmacytoma variant translocation 1; SC, scrambled; shRNA, short hairpin RNA; siRNA, small interfering RNA.

$(\mathrm{P}<0.05)$. Additionally, no significant differences between the LV-NC-shRNA group and the blank control cell group were identified $(\mathrm{P}>0.05)$.

The protein expression levels of c-Myc were assessed by western blotting. As shown in Fig. 1C, western blot analysis confirmed that c-Myc protein expression was decreased in the Raji cells transfected with PVT1 siRNA1055 after $48 \mathrm{~h}$ compared with in the SC siRNA and blank control cell groups. There were no clear differences in c-Myc expression among the SC siRNA group, mock-transfected cells and blank control cells. Similarly, c-Myc protein expression was decreased in Raji cells stably carrying LV-PVT1-shRNA compared with either the LV-NC-shRNA group or blank control group (Fig. 1D). There was no difference identified between c-Myc expression in the LV-NC-shRNA group and blank control group. These findings indicated that PVTI siRNA and shRNA were effective at reducing c-Myc protein expression.

Effect of PVT1 knockdown on the proliferation and cell cycle distribution of Raji cells. A CCK8 assay was performed to quantify cell proliferation. As shown in Fig. 2A, the proliferative activity of Raji cells carrying the LV-PVT1-shRNA was significantly inhibited $(\mathrm{P}<0.01)$ compared with Raji cells carrying LV-NC-shRNA and blank control cells $(\mathrm{P}<0.01)$. No significant difference in proliferation of LV-NC-shRNA group and blank control cells was identified. $P V T 1$ siRNA had a similar effect on the proliferative activity of Raji cells (data not shown).
Proliferation of Raji cells was inhibited following PVT1 knockdown. Additionally, the effect of decreased PVTI expression on the cell cycle was examined. According to flow cytometric analysis, the percentages of cells in $\mathrm{G}_{0} / \mathrm{G}_{1}, \mathrm{~S}$ and $\mathrm{G}_{2} / \mathrm{M}$ phases were $54.20 \pm 0.61,34.07 \pm 0.64$ and $11.70 \pm 0.00 \%$ in Raji cells carrying LV-PVT1-shRNA, respectively. In Raji cells carrying LV-NC-shRNA, the percentages of cells in $\mathrm{G}_{0} / \mathrm{G}_{1}, \mathrm{~S}$ and $\mathrm{G}_{2} / \mathrm{M}$ phases were $47.37 \pm 0.60,43.27 \pm 0.55$ and $9.37 \pm 0.51 \%$, respectively. There was a significant decrease in the percentage of cells in the $S$ phase and a marked accumulation of cells in the $G_{0} / G_{1}$ phase in Raji cells carrying LV-PVT1-shRNA compared with those carrying LV-NC-shRNA and blank control cells (Fig. 2B and C). There was no significant difference identified between the Raji cells carrying LV-NC-shRNA and blank control cells. These results indicated that knockdown of PVT1 expression in Raji cells induced $\mathrm{G}_{0} / \mathrm{G}_{1}$ phase arrest.

Alteration in expression pattern of cell cycle-associated genes in Raji cells as a result of PVT1 knockdown. Differential mRNA expression levels of 84 genes involved in the cell cycle were assessed. The results of the PCR array revealed that 54 genes were upregulated and 26 genes were downregulated in the LV-PVT1-shRNA group compared with the LV-NC-shRNA group. The other four genes exhibited too low an expression level. There were more upregulated genes than downregulated genes in Raji cells carrying LV-PVT1-shRNA (Fig. 3A; Table II). The 

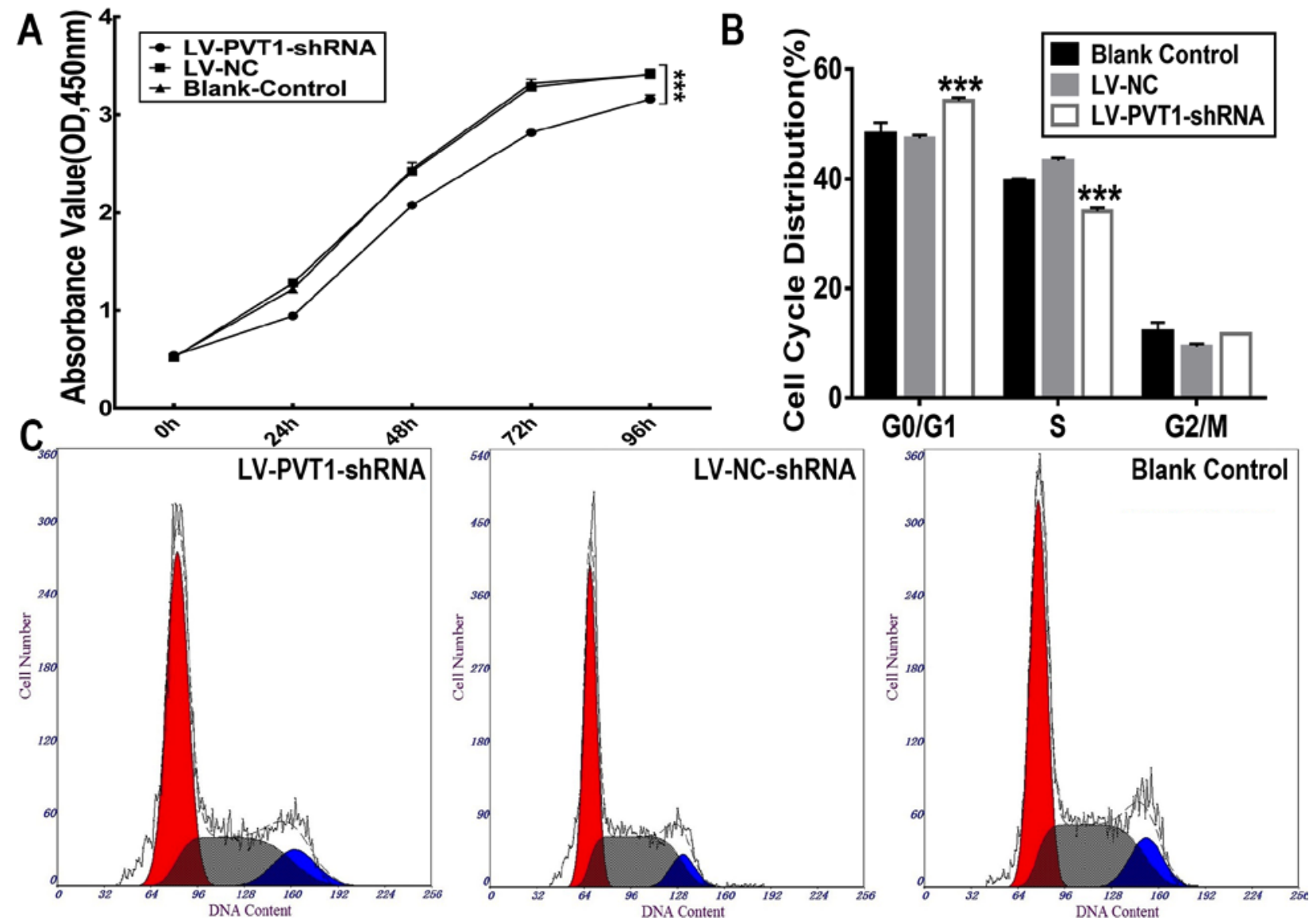

Figure 2. Effect of PVT1 knockdown on the proliferation and cell cycle distribution of Raji cells. (A) Raji cell proliferation was determined by Cell Counting kit-8 assay. Raji cells stably infected with LV-PVT1-shRNA and LV-NC-shRNA were incubated for different time intervals (24, 48, 72 and 96 h). Absorbance values were plotted. (B) Cell cycle distribution of Raji cells stably infected with LV-PVT1-shRNA was analysed by flow cytometry. The results represent the average values of three independent experiments \pm standard deviation. There was a significant decrease in the percentage of cells in the $\mathrm{S}$ phase and a marked accumulation of cells in the $\mathrm{G}_{0} / \mathrm{G}_{1}$ phase in Raji cells carrying LV-PVT1-shRNA compared with those carrying LV-NC-shRNA and blank control cells. (C) Representative fluorescence-activated cell sorting images of cell cycle distribution analysis of Raji cells. ${ }^{* * *} \mathrm{P}<0.01$ vs. LV-NC-shRNA. LV, lentiviral vector; NC, negative control; OD, optical density; $P V T 1$, plasmacytoma variant translocation 1; shRNA, short hairpin RNA.

present study demonstrated that $16 / 84$ examined genes were upregulated at least two-fold in the LV-PVT1-shRNA group.

Candidate gene selected from PCR microarray analyses was validated. As shown in Fig. 3B, western blotting revealed that $\mathrm{P} 21$, encoded by cyclin-dependent kinase inhibitor $1 \mathrm{~A}$ $(C D K N 1 A)$ was increased and CCNE1 was decreased in the LV-PVT1-shRNA group compared within the LV-NC-shRNA group and in blank control cells. As shown in Fig. 3C, the RT-qPCR assay demonstrated that the expression levels of cyclin G2 (CCNG2), Retinoblastoma-like 2 (RBL2, p130), CDKN1A, HUS1 checkpoint homolog (HUS1), cyclin dependent kinase inhibitor $3(C D K N 3)$ and cyclin dependent kinase inhibitor $1 \mathrm{~B}(C D K N 1 B)$ were upregulated in Raji cells infected with LV-PVT1-shRNA compared with the LV-NC-shRNA group and blank control cells. Although the expression of $C D K N 1 B$ was very low in the three groups, there was statistically significant difference between the LV-PVT1-shRNA group and the LV-NC-shRNA group $(\mathrm{P}<0.05)$. In addition, $C C N E 1$, $C C N D 1$ and cell division cycle $20(C D C 20)$ were significantly downregulated in the LV-PVT1-shRNA group compared with in the LV-NC-shRNA group and blank control cells $(\mathrm{P}<0.05)$. The RT-qPCR assay results of the 9 selected differentially expressed genes did not change notably compared with the PCR array results.

\section{Discussion}

PVT1 is aberrantly expressed in a variety of tumour types and acts as a potential oncogene that promotes cancer cell proliferation (8-12). Gain of $P V T 1$ lncRNA expression is required for high MYC protein levels in 8q24-amplified human cancer cells (13). PVT1 RNA and MYC protein expression are correlated in primary human tumours, and copy number of PVT1 co-increases in $>98 \%$ of MYC-copy-increase types of cancer (13). Although it was reported in 1990 that the PVT1 locus may be a site of variant translocations, including in human $\mathrm{BL}$ (6), to the best of our knowledge, there are no reports about the role of PVT1 in BL. To investigate the role of PVT1 in BL cell proliferation, RNA interference was used to inhibit its expression in the human BL-derived Raji cell line. The results of the present study revealed that the expression levels of PVT1 RNA and c-Myc protein decreased following transfection of Raji cells with $P V T 1$ siRNA targeting the 1,055-1,074 nt region of the PVT1 sequence. No significant effect of the other three siRNAs targeting $P V T 1$ (siRNA54, siRNA176 and siRNA845) on the expression levels of PVT1 RNA was identified. The specific siRNA against PVT1, siRNA1055, was successfully incorporated into a lentiviral vector. Raji cells carrying the PVT1-shRNA lentiviral vector were screened. Once Raji cells 


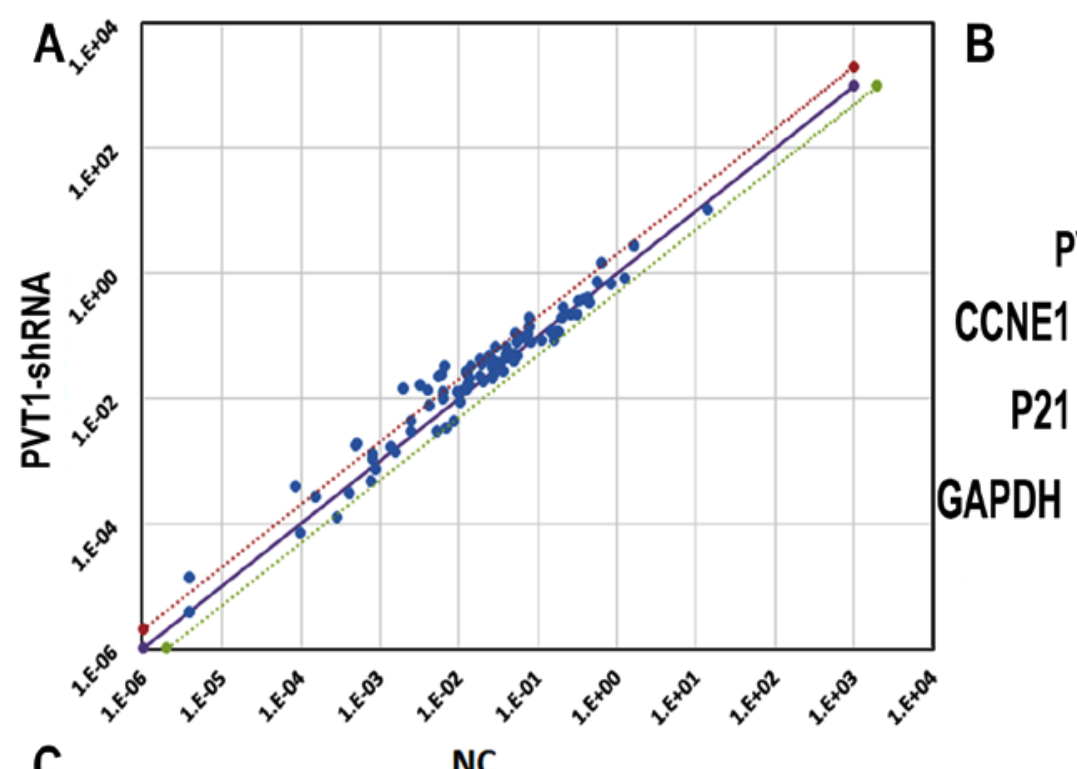

\section{PVT1-shRNA NC Blank Control}

CCNE1

P21
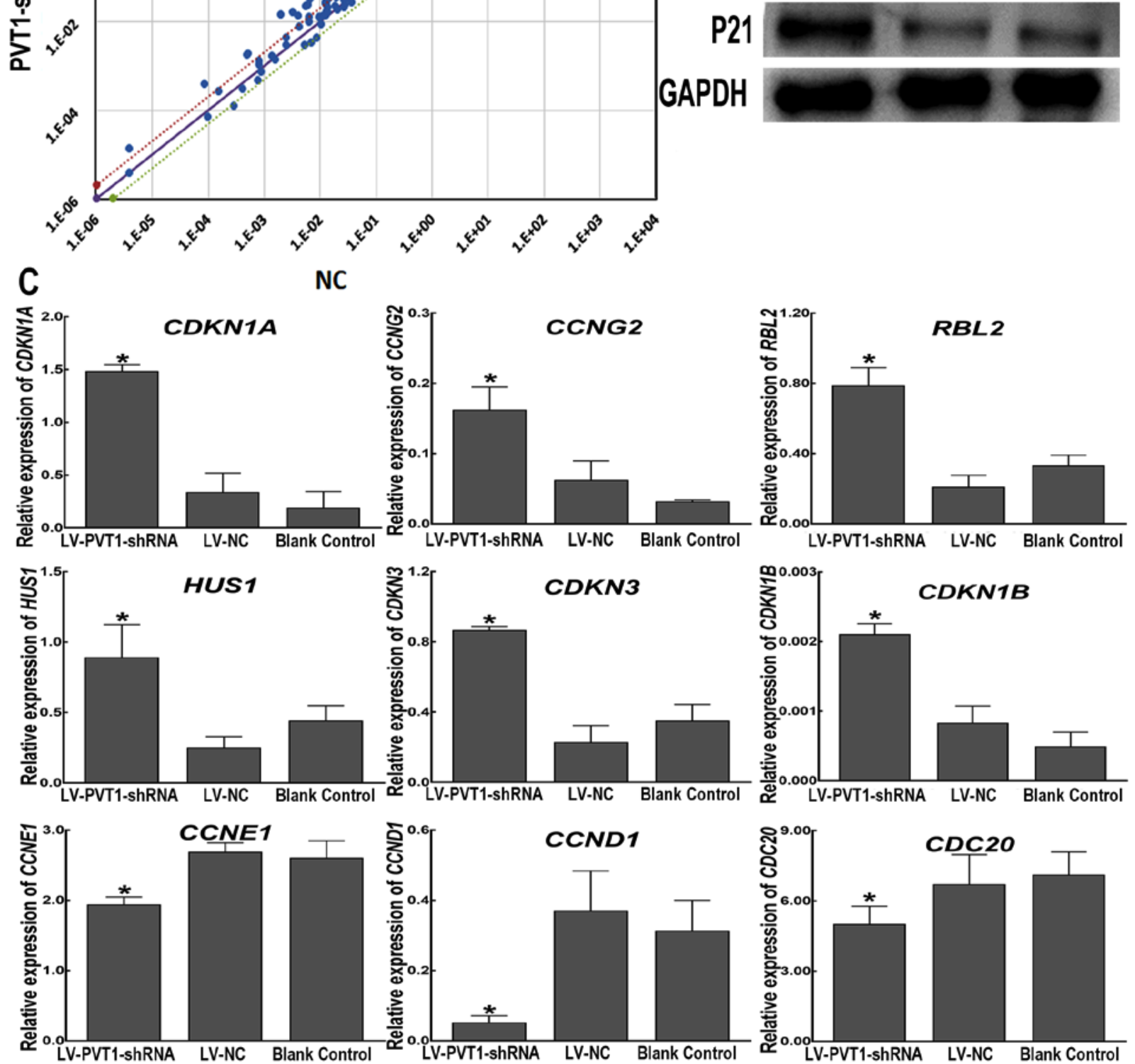

Figure 3. Differential expression of cell cycle-associated genes in Raji cells with PVT1 knockdown. (A) There are three straight lines in the coordinate system, and the black line represents the fold changes $\left(2^{-\Delta \Delta C t}\right)$ of 1 . Dots above the black line represent genes that are upregulated, and dots below the black line indicate genes that are downregulated in the LV-PVT1-shRNA group compared with the LV-NC-shRNA group. A point within the range of the two dotted lines signifies a differential expression of $>1$-fold but $<2$-fold in Raji cells infected with LV-PVT1-shRNA compared with cells infected with LV-NC-shRNA. Points outside the two dotted lines are $>2$-fold differentially expressed. (B) Protein expression levels of P21 and CCNE1 in Raji cells were determined using western blot analysis following $P V T 1$ knockdown. GAPDH served as a loading control. (C) Reverse transcription-quantitative polymerase chain reaction analysis of nine genes in Raji cells stably infected with LV-PVT1-shRNA compared with the genes in the negative control cells infected with LV-NC-shRNA. GAPDH was used as a reference gene. The graphs depict the mean mRNA expression changes \pm standard deviation of three independent experiments. ${ }^{*} \mathrm{P}<0.05$ vs. LV-NC-shRNA. CCNE1, cyclin E1; CCND1, cyclin D1; CCNG2, cyclin G2; CDC20, cell division cycle 20 homolog (S. cerevisiae); CDKN1A, cyclin-dependent kinase inhibitor 1A (P21, Cip1); $C D K N 1 B$, cyclin-dependent kinase inhibitor 1B (P27, Kip1); CDKN3, cyclin-dependent kinase inhibitor 3; HUS1, HUS1 checkpoint homolog (S. pombe); LV, lentiviral vector; NC, negative control; $P V T 1$, plasmacytoma variant translocation 1; RBL2, Retinoblastoma-like 2 (p130); shRNA, short hairpin RNA.

stably expressed $P V T 1$-shRNA, the levels of $P V T 1$ RNA and c-Myc protein were markedly reduced, which is consistent with the effect of $P V T 1$ siRNA1055.
In the present study, the proliferation of Raji cells carrying $P V T 1$-shRNA was significantly decreased compared with in control cells. However, downregulation of $P V T 1$ expression 
Table II. Differentially expressed genes associated with the cell cycle in Raji cells followingPVT1 knockdown.

\begin{tabular}{|c|c|c|c|}
\hline Gene symbol & Accession no. & Gene description & Fold change \\
\hline CCNG2 & NM_004354 & Cyclin G2 & 7.03 \\
\hline$R B L 2$ & NM_005611 & Retinoblastoma-like 2 (p130) & 5.26 \\
\hline$C D K N 1 A$ & NM_000389 & Cyclin-dependent kinase inhibitor 1A (P21, Cip1) & 4.78 \\
\hline CCNT1 & NM_001240 & Cyclin T1 & 4.17 \\
\hline CASP3 & NM_004346 & Caspase 3, apoptosis-related cysteine peptidase & 3.96 \\
\hline$R B L 1$ & NM_002895 & Retinoblastoma-like 1 (p107) & 3.70 \\
\hline$R B 1$ & NM_000321 & Retinoblastoma 1 & 3.68 \\
\hline HUS1 & NM_004507 & HUS1 checkpoint homolog (S. pombe) & 3.38 \\
\hline CCND2 & NM_001759 & Cyclin D2 & 2.51 \\
\hline CDC6 & NM_001254 & Cell division cycle 6 homolog (S. cerevisiae) & 2.34 \\
\hline$C D K N 1 B$ & NM_004064 & Cyclin-dependent kinase inhibitor 1B (p27, Kip1) & 2.31 \\
\hline$T F D P 2$ & NM_006286 & Transcription factor Dp-2 (E2F dimerization partner 2) & 2.23 \\
\hline$C D C 16$ & NM_003903 & Cell division cycle 16 homolog (S. cerevisiae) & 2.21 \\
\hline$G A D D 45 A$ & NM_001924 & Growth arrest and DNA-damage-inducible, alpha & 2.08 \\
\hline CDKN3 & NM_005192 & Cyclin-dependent kinase inhibitor 3 & 2.07 \\
\hline$A B L 1$ & NM_005157 & C-abl oncogene 1 , non-receptor tyrosine kinase & 2.04 \\
\hline$B R C A 2$ & NM_000059 & Breast cancer 2 , early onset & -2.22 \\
\hline CCNE1 & NM_001238 & Cyclin E1 & -2.05 \\
\hline CCND1 & NM_053056 & Cyclin D1 & -1.98 \\
\hline CDK6 & NM_001259 & Cyclin-dependent kinase 6 & -1.85 \\
\hline$C D C 20$ & NM_001255 & Cell division cycle 20 homolog (S. cerevisiae) & -1.81 \\
\hline$A T M$ & NM_000051 & Ataxia telangiectasia mutated & -1.67 \\
\hline$C K S 2$ & NM_001827 & CDC28 protein kinase regulatory subunit 2 & -1.56 \\
\hline$C D K 4$ & NM_000075 & Cyclin-dependent kinase 4 & -1.52 \\
\hline
\end{tabular}

Positive and negative values represent upregulation or downregulation of genes in the LV-PVT1-shRNA group compared with the LV-NC-shRNA group, respectively.

by shRNA could not induce apoptosis of Raji cells (data not shown). Cell cycle distribution analysis indicated that knockdown of PVT1 in Raji cells resulted in notable $\mathrm{G}_{0} / \mathrm{G}_{1}$ phase arrest. Previous studies have reported that PVT1 can promote the proliferation of cells, including hepatocellular carcinoma (10) and thyroid cancer cells (11). The results of the present study were consistent with those of previous studies. Taken together, the results suggested that the PVTI shRNA-mediated suppression of Raji cell proliferation may occur via cell cycle arrest.

PVT1 may be associated with a series of signalling pathways and genes in tumour development and progression (18-20). According to topological measures, Paci et al (20) revealed that the lncRNA $P V T 1$ is connected to 753 different mRNAs ( $\sim 50 \%$ of total mRNAs in the network), and the miR-200 family members mediate $>80 \%$ of these interactions. However, the mechanism by which knockdown of PVT1 causes cell cycle alteration in Raji cells remains largely unexplained. It has been confirmed that $P V T 1$ is located adjacent to the proto-oncogene $c-M y c$, and the expression of $P V T 1-M y c$ in the majority of tumours is positively correlated with the copy number of $8 \mathrm{q} 24(13,15,21)$. Tseng et al $(13)$ demonstrated that c-Myc protein expression is dependent on the expression levels of PVT1; therefore, this IncRNA may promote cell proliferation and tumourigenesis by regulating the expression of c-Myc protein. Following transfection of Raji cells with $P V T 1$-siRNA or shRNA, the expression of c-Myc protein decreased, which was consistent with previous reports (13). It has been demonstrated that c-Myc is involved in regulation of the cell cycle and proliferation (22). Yang et al (23) revealed that c-Myc regulates the cyclin-dependent kinasel (CDK1)/cyclin B1-dependent $\mathrm{G}_{2} / \mathrm{M}$ cell cycle progression by controlling histone $\mathrm{H} 4$ acetylation. Additionally, a study has reported that loss of c-Myc function impedes $\mathrm{G}_{1}$-phase progression (22). The CDK inhibitors P27 and P21 appear to be critical targets of c-Myc, which cooperates with the zinc finger MIZ-type containing 1 to repress the transcription of cell-cycle inhibitors, including P15, P21 and P27 (24). Yang et al (23) also reported that suppression of c-Myc induces the upregulation of P21 and the downregulation of P27 in Raji cells. Recently, Wang et al (25) reported that the epigenetically induced lncRNA EPIC1 promotes cell cycle progression by interacting with c-Myc via the 129-283 nt region of EPIC1. EPICl knockdown reduces the affinity of c-Myc to its target genes, including CDKN1A, CCNA2, CDC20 and $C D C 45$ (25). The results of the present study suggested that the regulation of $\mathrm{G}_{0} / \mathrm{G}_{1}$ cell cycle progression by PVT1 may be associated with c-Myc expression or c-Myc regulation of cell cycle-associated genes. 
To further evaluate the alterations in cell cycle-associated gene expression in Raji cells with $P V T 1$ knockdown, a cell cycle PCR microarray was used to detect alterations in cell cycle-associated genes in Raji cells stably expressing $P V T 1$-shRNA. The results revealed that cell cycle-associated genes, including $C C N G 2, R B L 2, C D K N 1 A, H U S 1, C D K N 3$ and $C D K N 1 B$, were upregulated in Raji cells with $P V T 1$ knockdown. The majority of the aforementioned genes inhibit cell cycle progression. CDKN1A encodes a cell cycle-dependent kinase inhibitor, which binds and inhibits the activity of cell cycle-associated genes with cyclin and CDK complexes, including CCND1-CDK4 and CCNE-CDK2. $\mathrm{P} 21$ is a member of the cell cycle regulator CIP/KIP family, which is involved in the regulation of numerous important biological behaviours, the most important of which is the regulation of cell cycle progression, particularly cell cycle arrest at the $\mathrm{G}_{1}$ phase $(26,27)$. Therefore, P21 serves a key role in cell quiescence, senescence and differentiation (28). In addition, a recent study identified a positive correlation between $P V T 1$ and miR-1207-5P, and a negative correlation between miR-1207-5P and signal transducer and activator of transcription 6 (STAT6) in breast cancer (29). miR-1207-5P, produced by PVT1 transcription and targeting STAT6, promotes the proliferation of breast cancer cells by regulating P21 and CDKN1B (29). In nasopharyngeal cancer cells, $P V T 1$ promotes cancer stem cell-like properties by inhibiting miR-1207 and activating the phosphoinositide 3-kinase/protein kinase B signalling pathway (30). In the present study, following the downregulation of $P V T I$ expression in Raji cells, the RNA expression levels of CDKN1A and P21 protein expression were increased, and the cell cycle was blocked in $\mathrm{G}_{0} / \mathrm{G}_{1}$ phase, suggesting that $P V T 1$ may promote cell cycle progression by inhibiting the expression of $\mathrm{P} 21$. In pancreatic cancer cell lines, $P V T l$ can promote epithelial-to-mesenchymal transition by downregulating the expression of P21, thus promoting cell proliferation and migration (31). In addition, a study by Cui et al (32) suggested that PVTI promotes cell proliferation and migration by downregulating P21 in pancreatic cancer cells. Therefore, the results of the present study were consistent with the literature.

$C C N G 2$, which has been shown to be associated with various types of tumour, can cause cell cycle arrest in the $\mathrm{G}_{1}$ phase (33-37). A study by Cui et al (37) indicated that $C C N G 2$ expression is decreased in prostate cancer and that PC-3 cells transfected with $C C N G 2$ exhibit a lower survival rate, a higher percentage of cells in the $\mathrm{G}_{0} / \mathrm{G}_{1}$ phases and lower CDK2 protein expression, suggesting that $C C N G 2$ may serve important roles as a negative regulator of prostate cancer cells. The results of the present study demonstrated that the downregulation of $P V T 1$ in Raji cells increased the expression of $C C N G 2$, which suggested that $P V T 1$ may also promote cell cycle progression by inhibiting the expression of $C C N G 2$. The RBL2 gene, encoding the proline rich protein BstNI subfamily $2(\mathrm{pRb} 2)$ protein, is most abundant in the $\mathrm{G}_{0}$ phase. $R B L 2$ maintains $\mathrm{G}_{0}$ arrest in quiescent or differentiated cells, controls the transition from $G_{1}$ to $S$ phase, and is a key regulator of growth arrest in cellular senescence (38). AKT inhibition reduces cell viability, induces cell accumulation in $\mathrm{G}_{0} / \mathrm{G}_{1}$ phase and triggers apoptosis, which proves to be largely dependent on RBL2/pRb2 itself, as shown by RBL2/pRb2 silencing (39). The HUS1 protein, encoded by the HUS1 gene, is a component of an evolutionarily conserved and genotoxin-activated checkpoint complex that is involved in cell cycle arrest in response to DNA damage. In the present study, the expression levels of the cell cycle progression regulators RBL2 and HUS1 were increased in Raji cells with $P V T 1$ knockdown, suggesting that the involvement of $P V T 1$ in cell cycle progression may be associated with the expression of RBL2 and HUS1. CDKN3 (also called CDI1 or KAP), encoded by the CDKN3 gene, is a member of the dual specificity protein phosphatase family, which is essential for mitosis and $\mathrm{G}_{1} / \mathrm{S}$ phase transition, due to its role in regulating the CDK1 (also called CDC2) signalling axis (40), and $C D K N 3$ serves an important role in regulating cell division. $C D K N 3$ may also function as an oncogene by inducing $\mathrm{G}_{0} / \mathrm{G}_{1}$ phase progression, apoptosis and metastasis in ovarian cancer cell lines (41). In the present study, the expression levels of $C D K N 3$ were increased in Raji cells with $P V T 1$ knockdown, suggesting that $C D K N 3$ may be involved in the cell cycle arrest by the induction of $P V T I$ downregulation.

In the present study, $C C N E 1, C C N D 1$ and $C D C 20$ were significantly downregulated in Raji cells following knockdown of PVT1. CCNE1 is a positive regulator of the cell cycle that controls the transition of cells from the $G_{1}$ to the $S$ phase, and serves an important role in cell proliferation and tumourigenesis. Overexpression of $C C N E 1$ can increase tumour incidence and susceptibility to multiple tumourigenesis in mice (42-44). $C C N D 1$, which belongs to the $\mathrm{G}_{1}$ cyclins, serves an important role in cell cycle regulation and promotes cell cycle progression from the $\mathrm{G}_{1}$ phase to $\mathrm{S}$ phase through its interaction with CDKs. CCNDI is overexpressed and/or amplified in numerous human types of cancer $(45,46)$. A previous study reported that CCND1 allows for the progression from the $\mathrm{G}_{1}$ to the $\mathrm{S}$ phase by binding and sequestering P21 (47). A study by Li et al (48) suggested that $P V T 1$ promotes cell cycle progression by increasing the expression of $C C N D 1$. The mechanism involved indicated that $P V T 1$ promotes the progression of clear cell renal cell carcinoma partly via activation of the epidermal growth factor receptor pathway (48). In melanoma cells, silencing of $P V T 1$ significantly inhibits cell proliferation, migration and invasion, and arrests the cell cycle at $\mathrm{G}_{0} / \mathrm{G}_{1}$ stage by significantly decreasing $C C N D 1$ expression (49). CDC20, which has important functions in chromosome segregation and mitotic exit, is abnormally expressed in a wide range of tumours, including human bladder carcinoma, pancreatic, colorectal breast and lung cell cancer (50). It has been reported that $\mathrm{CDC} 20$ were significantly downregulated by lncRNA EPIC1 knockdown in both tumor samples and cell lines (25). Therefore, the results of the present study suggested that the role of $P V T I$ in regulating $\mathrm{G}_{0} / \mathrm{G}_{1}$ cell cycle progression was likely to be associated with the expression levels of CCNE1, CCND1 and CDC20.

In conclusion, the results of the present study revealed that knockdown of $P V T 1$ may inhibit the proliferation of Raji cells by arresting cells in the $\mathrm{G}_{0} / \mathrm{G}_{1}$ phase. The role of $P V T 1$ in regulating cell cycle progression may be associated with the expression of c-Myc and cell cycle-associated genes, including $C C N G 2, R B L 2$, CDKN1A, HUS1, CDKN3, CDKN1B, CCNE1, CCND1 and $C D C 20$. However, it remains unclear how $P V T 1$ influences the expression levels of cell cycle-associated genes. Furthermore, it is not clear which signalling pathways could be directly associated with $P V T 1$. Therefore, further studies are required to understand the potential molecular mechanisms that underlie the regulation 
of cell cycle-associated genes by $P V T 1$ in Raji cells. Certainly, the expression pattern of PVT1 in BL should be investigated following the collection of clinical samples. It will be useful to develop a comprehensive understanding of the role of $P V T 1$ and its potential as an oncotarget in BL.

\section{Acknowledgements}

The authors would like to thank associate Professor Gexiu Liu and senior experimentalist Mrs Shaohua Chen, School of Medicine, Jinan University, Guangzhou, for providing valuable technical support and advice.

\section{Funding}

The project was supported by grants from the Guangdong Science and Technology Project (grant no. 2015A050502029) and the Overseas Chinese Affairs Office of the State Council Key Discipline Construction Fund (grant no. 51205002).

\section{Availability of data and materials}

The datasets used and/or analysed in the present study are available from the corresponding author on reasonable request.

\section{Authors' contributions}

$\mathrm{CZ}$ and $\mathrm{YX}$ performed the experiments and interpreted results. DH and YL developed the original concept, designed the study and contributed to the writing of the manuscript.

\section{Ethics approval and consent to participate}

Not applicable.

\section{Patient consent for publication}

Not applicable.

\section{Competing interests}

The authors declare that they have no competing interests.

\section{References}

1. Molyneux EM, Rochford R, Griffin B, Newton R, Jackson G, Menon G, Harrison CJ, Israels T and Bailey S: Burkitt's lymphoma. Lancet 379: 1234-1244, 2012.

2. Boxer LM and Dang CV: Translocations involving c-myc and c-myc function. Oncogene 20: 5595-5610, 2001.

3. Fang Y and Fullwood MJ: Roles, functions, and mechanisms of long non-coding RNAs in cancer. Genomics Proteomics Bioinformatics 14: 42-54, 2016.

4. Dykes IM and Emanueli C: Transcriptional and post-transcriptional gene regulation by long non-coding RNA. Genomics Proteomics Bioinformatics 15: 177-186, 2017.

5. Rodríguez-Malavé NI and Rao DS: Long noncoding RNAs in hematopoietic malignancies. Brief Funct Genomics 15: 227-238, 2016.

6. Shtivelman E and Bishop JM: Effects of translocations on transcription from PVT. Mol Cell Biol 10: 1835-1839, 1990.

7. Huppi K and Siwarski D: Chimeric transcripts with an open reading frame are generated as a result of translocation to the Pvt-1 region in mouse B-cell tumors. Int J Cancer 59: 848-851, 1994.
8. Wang BJ, Ding HW and Ma GA: Long noncoding RNA PVT1 promotes melanoma progression via endogenous sponging miR-26b. Oncol Res 26: 675-681, 2018.

9. Ding C, Yang Z, Lv Z, Du C, Xiao H, Peng C, Cheng S, Xie H, Zhou L, Wu J and Zheng S: Long non-coding RNA PVT1 is associated with tumor progression and predicts recurrence in hepatocellular carcinoma patients. Oncol Lett 9: 955-963, 2015.

10. Wang F, Yuan JH, Wang SB, Yang F, Yuan SX, Ye C, Yang N, Zhou WP, Li WL, Li W and Sun SH: Oncofetal long noncoding RNA PVT1 promotes proliferation and stem cell-like property of hepatocellular carcinoma cells by stabilizing NOP2 Hepatology 60: 1278-1290, 2014.

11. Zhou Q, Chen J, Feng J and Wang J: Long noncoding RNA PVT1 modulates thyroid cancer cell proliferation by recruiting EZH2 and regulating thyroid-stimulating hormone receptor (TSHR). Tumour Biol 37: 3105-3113, 2016.

12. Takahashi Y, Sawada G, Kurashige J, Uchi R, Matsumura T, Ueo H, Takano Y, Eguchi H, Sudo T, Sugimachi K, et al: Amplification of PVT-1 is involved in poor prognosis via apoptosis inhibition in colorectal cancers. Brit J Cancer 110: 164-171, 2014.

13. Tseng YY, Moriarity BS, Gong W, Akiyama R, Tiwari A, Kawakami H, Ronning $\mathrm{P}$, Reuland $\mathrm{B}$, Guenther $\mathrm{K}$, Beadnell TC, et al: PVT1 dependence in cancer with MYC copy-number increase. Nature 512: 82-86, 2014.

14. Huang F, Chen W, Peng J, Li Y, Zhuang Y, Zhu Z, Shao C, Yang W, Yao $\mathrm{H}$ and Zhang S: LncRNA PVT1 triggers Cyto-protective autophagy and promotes pancreatic ductal adenocarcinoma development via the miR-20a-5p/ULK1 Axis. Mol Cancer 17: 98, 2018.

15. Ghesquières $\mathrm{H}$, Larrabee $\mathrm{BR}$, Casasnovas $\mathrm{O}$, Maurer $\mathrm{MJ}$, McKay JD, Ansell SM, Montgomery D, Asmann YW, Farrell K, Verney A, et al: A susceptibility locus for classical Hodgkin lymphoma at 8q24 near MYC/PVT1 predicts patient outcome in two independent cohorts. Brit J Haematol 180: 286-290, 2018.

16. Cerhan JR, Berndt SI, Vijai J, Ghesquières H, McKay J, Wang SS, Wang Z, Yeager M, Conde L, de Bakker PI, et al: Genome-wide association study identifies multiple susceptibility loci for diffuse large B cell lymphoma. Nat Genet 46: 1233-1238, 2014.

17. Livak KJ and Schmittgen TD: Analysis of relative gene expression data using real-time quantitative PCR and the 2 (-Delta Delta C(T)) method. Methods 25: 402-408, 2001.

18. Zhang Y, Mo WJ, Wang X, Zhang TT, Qin Y, Wang HL, Chen G, Wei DM and Dang YW: Microarray-based bioinformatics analysis of the prospective target gene network of key miRNAs influenced by long non-coding RNA PVT1 in HCC. Oncol Rep 40: 226-240, 2018.

19. Zhang Y, Dang YW, Wang X, Yang X, Zhang R, Lv ZL and Chen G: Comprehensive analysis of long non-coding RNA PVT1 gene interaction regulatory network inhepatocellular carcinoma using gene microarray and bioinformatics. Am J Transl Res 9: 3904-3917, 2017.

20. Paci P, Colombo T and Farina L: Computational analysis identifies a sponge interaction network between long non-coding RNAs and messenger RNAs in human breast cancer. BMC Syst Biol 8: 83, 2014

21. Kong R, Zhang EB, Yin DD, You LH, Xu TP, Chen WM, Xia R, Wan L, Sun M, Wang ZX, et al: Long noncoding RNA PVT1 indicates a poor prognosis of gastric cancer and promotes cell proliferation through epigenetically regulating p15 and p16. Mol Cancer 14: 82, 2015.

22. Bretones G, Delgado MD and León J: Myc and cell cycle control. Biochim Biophys Acta 1849: 506-516, 2015.

23. Yang Y Xue K, Li Z, Zheng W, Dong W, Song J, Sun S, Ma T and Li W: c-Myc regulates the CDK1/cyclin B1 dependent-G2/M cell cycle progression by histone $\mathrm{H} 4$ acetylation in Raji cells. Int J Mol Med 41: 3366-3378, 2018.

24. Dang CV: MYC on the path to cancer. Cell 149: 22-35, 2012.

25. Wang Z, Yang B, Zhang M, Guo W, Wu Z, Wang Y, Jia L, Li S, Cancer Genome Atlas Research Network, Xie W and Yang D: lncRNA epigenetic landscape analysis identifies EPIC1 as an oncogenic lncRNA that interacts with MYC and promotes cell-cycle progression in cancer. Cancer Cell 33: 706-720.e9, 2018.

26. Dutto I, Tillhon M, Cazzalini O, Stivala LA and Prosperi E: Biology of the cell cycle inhibitor p21 (CDKN1A): Molecular mechanisms and relevance in chemical toxicology. Arch Toxicol 89: 155-178, 2015.

27. Cazzalini O, Scovassi AI, Savio M, Stivala LA and Prosperi E: Multiple roles of the cell cycle inhibitor p21 (CDKN1A) in the DNA damage response. Mutat Res 704: 12-20, 2010. 
28. Perucca P, Cazzalini O, Madine M, Savio M, Laskey RA, Vannini V, Prosperi E and Stivala LA: Loss of p21 CDKN1A impairs entry to quiescence and activates a DNA damage response in normal fibroblasts induced to quiescence. Cell Cycle 8: 105-114, 2009.

29. Yan C, Chen Y, Kong W, Fu L, Liu Y, Yao Q and Yuan Y: PVT1-derived miR-1207-5p promotes breast cancer cell growth by targeting STAT6. Cancer Sci 108: 868-876, 2017.

30. Cui M, Chang Y, Fang QG, Du W, Wu JF, Wang JH, Liu ST and Luo SX: Non-coding RNA Pvt1 promotes cancer stem cell-like traits in nasopharyngeal cancer via inhibiting miR-1207. Pathol Oncol Res, 2018.

31. Wu BQ, Jiang Y, Zhu F, Sun DL and He XZ: Long noncoding RNA PVT1 promotes EMT and cell proliferation and migration through downregulating p21 in pancreatic cancer cells. Technol Cancer Res Treat 1: 1533034617700559, 2017.

32. Cui D, Yu CH, Liu M, Xia QQ, Zhang YF and Jiang WL: Long non-coding RNA PVT1 as a novel biomarker for diagnosis and prognosis of non-small cell lung cancer. Tumor Biol 37: 4127-4134, 2016

33. Bennin DA, Don AS, Brake T, McKenzie JL, Rosenbaum H, Ortiz L, DePaoli-Roach AA and Horne MC: Cyclin G2 associates with protein phosphatase $2 \mathrm{~A}$ catalytic and regulatory B' subunits in active complexes and induces nuclear aberrations and a G1/S phase cell cycle arrest. J Biol Chem 277: 27449-27467, 2002.

34. Hasegawa S, Nagano H, Konno M, Eguchi H, Tomokuni A, Tomimaru Y, Wada H, Hama N, Kawamoto K, Kobayashi S, et al: Cyclin G2: A novel independent prognostic marker in pancreatic cancer. Oncol Lett 10: 2986-2990, 2015.

35. Yin G, Zhou H, Xue Y, Yao B and Zhao W: MicroRNA-340 promotes the tumor growth of human gastric cancer by inhibiting cyclin G2. Oncol Rep 36: 1111-1118, 2016.

36. Zimmermann M, Arachchige-Don AP, Donaldson MS Patriarchi T and Horne MC: Cyclin G2 promotes cell cycle arrest in breast cancer cells responding to fulvestrant and metformin and correlates with patient survival. Cell Cycle 15: 3278-3295, 2016.

37. Cui DW, Cheng YJ, Jing SW and Sun GG: Effect of cyclin G2 on proliferative ability of prostate cancer PC-3 cell. Tumour Biol 35: 3017-3024, 2014.

38. Helmbold $\mathrm{H}$, Galderisi $\mathrm{U}$ and Bohn W: The switch from $\mathrm{pRb} / \mathrm{p} 105$ to Rb2/p130 in DNA damage and cellular senescence. J Cell Physiol 227: 508-513, 2012.

39. Pentimalli F, Forte IM, Esposito L, Indovina P, Iannuzzi CA, Alfano L, Costa C, Barone D, Rocco G and Giordano A: $\mathrm{RBL} 2 / \mathrm{p} 130$ is a direct AKT target and is required to induce apoptosis upon AKT inhibition in lung cancer and mesothelioma cell lines. Oncogene 37: 3657-3671, 2018.
40. Nalepa G, Barnholtz-Sloan J,Enzor R, Dey D, He Y, Gehlhausen JR, Lehmann AS, Park SJ, Yang Y, Yang X, et al: The tumor suppressor CDKN3 controls mitosis. J Cell Biol 201: 997-1012, 2013.

41. Yu C, Cao H, He X, Sun P, Feng Y, Chen L and Gong H: Cyclin-dependent kinase inhibitor 3 (CDKN3) plays a critical role in prostate cancer via regulating cell cycle and DNA replication signaling. Biomed Pharmacother 96: 1109-1118, 2017.

42. Bortner DM and Rosenberg MP: Induction of mammary gland hyperplasia and carcinomas in transgenic mice expressing human cyclin E. Mol Cell Biol 17: 453-459, 1997.

43. Ma Y, Fiering S, Black C, Liu X, Yuan Z, Memoli VA, Robbins DJ, Bentley HA, Tsongalis GJ, Demidenko E, et al: Transgenic cyclin $\mathrm{E}$ triggers dysplasia and multiple pulmonary adenocarcinomas. Proc Natl Acad Sci USA 104: 4089-4094, 2007.

44. Loeb KR, Kostner H, Firpo E, Norwood T, D Tsuchiya K, Clurman BE and Roberts JM: A mouse model for cyclin E-dependent genetic instability and tumorigenesis. Cancer Cell 8: 35-47, 2005.

45. Santarius T, Shipley J, Brewer D, Stratton MR and Cooper CS: A census of amplified and overexpressed human cancer genes. Nat Rev Cancer 10: 59-64, 2010.

46. Cheng G, Zhang L, Lv W, Dong C, Wang Y and Zhang J: Overexpression of cyclin D1 in meningioma is associated with malignancy grade and causes abnormalities in apoptosis, invasion and cell cycle progression. Med Oncol 32: 439, 2015.

47. Hall M, Bates S and Peters G: Evidence for different modes of action of cyclin-dependent kinase inhibitors: p15 and p16 bind to kinases, p21 and p27 bind to cyclins. Oncogene 11: 1581-1588, 1995.

48. Li W, Zheng Z, Chen H, Cai Y and Xie W: Knockdown of long non-coding RNA PVT1 induces apoptosis and cell cycle arrest in clear cell renal cell carcinoma through the epidermal growth factor receptor pathway. Oncol Lett 15: 7855-7863, 2018.

49. Chen L, Ma D, Li Y, Li X, Zhao L, Zhang J and Song Y: Effect of long non-coding RNA PVT1 on cell proliferation and migration in melanoma. Int J Mol Med 41: 1275-1282, 2018.

50. Kapanidou M, Curtis NL and Bolanos-Garcia VM: Cdc20: At the crossroads between chromosome segregation and mitotic exit. Trends Biochem Sci 42: 193-205, 2017.

(i) (-) This work is licensed under a Creative Commons Attribution-NonCommercial-NoDerivatives 4.0 International (CC BY-NC-ND 4.0) License. 